The results were compared with a cohort of white Northern European IBD patients to determine if there is interethnic variation in the minor allele frequency for the disease cohorts.

Methods IBD patients were recruited from the IBD clinics of five hospitals in North West London. Indian Asian patients with all four grandparents originating from South Asia and a confirmed diagnosis of IBD were included in the study. Patients and controls were genotyped using pyrosequencing and the results compared using the $\chi^{2}$ test.

Results 216 Indian Asian IBD patients (60 CD: $156 \mathrm{UC}$ ), 203 healthy Indian Asian controls and 146 white Northern European IBD patients (98 CD: 48UC) were recruited. All SNPs were in Hardy Weinberg equilibrium. There was no significant difference in the minor allele frequency (MAF) between Indian Asian IBD patients and ethnically matched controls for any of the 4 SNPs. However, the MAF for SNPs in IRGM and IL23R was significantly different between Indian Asian and European CD cohorts (IRGM rs13361189: 0.23 vs 0.11, $\mathrm{p}=0.004$; IRGM rs4958847, 0.28 vs $0.15 \mathrm{p}=0.005$; IL23R 0.8 vs $6.1, \mathrm{p}=0.02$ ).

Conclusion The polymorphisms of ATG16L1, IRGM and $I L 23 R$ that are associated with IBD in Western populations show no association with IBD in Indian Asians. These findings are similar to a Japanese study that showed these SNPs were not associated with CD in this ethnic group; however, one cannot exclude the possibility that an analysis of a larger sample size in the Indian Asian population might unveil such an association. The SNP frequencies in the South Asian IBD cohorts were significantly different to the European disease controls for SNPs in IRGM and IL23R showing that there are clear interethnic variations in risk allele frequencies.

Competing interests None.

Keywords genetics, Indian Asian.

\section{PTH-069 ETHNIC VARIATION IN THE FREQUENCY OF IBD RELATED POLYMORPHISIMS IN IRGM, ATG16L1 AND IL23R}

doi:10.1136/gut.2011.239301.470

D G Walker, ${ }^{1, *}$ A S Bancil, ${ }^{1}$ P S Rai, ${ }^{1}$ H R Williams, ${ }^{1}$ J Chambers, ${ }^{2}$ J S Kooner, ${ }^{3}$ T R Orchard ${ }^{1}{ }^{1}$ Gastroenterology, Imperial College London, London, UK; ${ }^{2}$ Epidemiology and Public Health, Imperial College London, London, UK; ${ }^{3}$ Cardiovascular Science, Imperial College London, London, UK

Introduction Recent genome wide association studies have identified a number of key single nucleotide polymorphisms (SNPs) that contribute to the susceptibility of inflammatory bowel disease (IBD). SNPs in the autophagy-related 16-like 1 gene (ATG16L1; rs2241880) and immunity-related GTPase family M gene (IRGM; rs13361189 and rs4958847) have been shown to confer susceptibility to Crohn's Disease (CD) in European/ North American populations. In addition, a SNP in the interleukin 23 Receptor (IL23R, rs11209026) has been shown to confer protection against both CD and ulcerative colitis (UC) in Caucasian patients. These findings are in contrast to studies from East Asia that have shown no association of these SNPs with inflammatory bowel disease (IBD). The aim was to investigate the prevalence of IBD related SNPs in ATG16L1, IRGM and IL23R in a cohort of South Asian IBD patients and controls. 